\title{
Autocuidado en el adulto mayor con diabetes tipo 2. Monterrey, México.
}

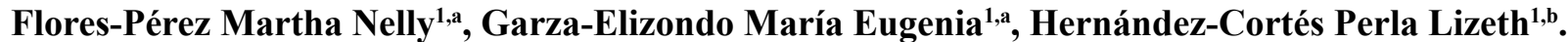

\section{RESUMEN}

Objetivos: determinar diferencias de autocuidado por edad y sexo en adultos mayores con diabetes tipo 2, que acuden a la consulta de medicina familiar. Material y métodos: estudio descriptivo de corte trasversal. La muestra estuvo constituida por 109 adultos mayores (63 Mujeres y 46 Hombres). Se aplicó el Resumen de Actividades de Autocuidado en Diabetes, se utilizó estadística descriptiva (frecuencias simples y relativas, medidas de dispersión e IC95\%), U de Mann-Whitney y diferencia de proporciones. Resultados: el promedio de tiempo de diagnóstico con la DT2 fue de 12,24 ( \pm 9.9$)$ años, el 31,2\% (34) comentaron tener alguna complicación propia de la DT2, la más común fue la retinopatía con un 22,9\% (25). El 33,0\% (36) de los adultos mayores tuvieron prácticas de autocuidado, las prácticas de cuidado que realizan con mayor frecuencia fueron cuidado de pies y consumo de medicamentos. El $28,6 \%$ (18) de las mujeres presentaron prácticas de autocuidado, esta cifra se reportó un poco mayor en los hombres $39,1 \%(18)(\mathrm{p}>0,05)$. Los adultos mayores de 60 a 69 años presentaron mayor prácticas de autocuidado que los mayores de 70 años $(34,9 \%$ vs 30,4\%; p > 0,05). Conclusiones: las prácticas de autocuidado en esta población fueron pobres a pesar de que tienen una patología, según las actividades se concluyó, que le dan mayor importancia al consumo de medicamentos y cuidado de los pies. Los hombres y los adultos mayores de menor edad son los que reportaron realizar mayor actividades de autocuidado.

PALABRAS CLAVE: Autocuidado, diabetes, adulto mayor.

\section{Self-care in older adults with type 2 diabetes. Monterrey, México.}

\section{SUMMARY}

Objectives: to determine self-care differences by age and gender in older adults with type 2 diabetes, attending in a family health clinic. Material and Methods: The sample was 109 older adults (63 women and 46 men). The self-care was evaluated with the Summary of Diabetes Self-Care Activities. The data analysis was used descriptive statistics (frequencies, measures of dispersion and 95\%CI), Mann-Whitney and difference of proportions. Results: The mean of diagnosis time was 12.24 ( \pm 9.9$)$ years, 31.2\% (34) said to have some complication by the pathology, the most common of this was retinopathy on $22.9 \%$ (25), 33.0\% (36) of older did self-care practices, the care that were performed more frequentlywerecare of foot and drugs; The women had self-care practices, this data was reported slightly higher in men $(28.6 \%$ vs $39.1 \%$; $p>0.05)$. Adults of $60-69$ years had higher self-care practices to those over 70 years $(4.9 \%$ vs $30.4 \%$; $>0.05)$. Conclusions: Self-care practices in this population are poor despite they have a disease, according to activities with higher frequencies we concludes that older give most importance to the consumption of drugs, and foot care. Men and younger older adults are reporting higher performing self-care activities.

KEYWORDS: Self-care, diabetes, older adults.

\footnotetext{
Universidad Autónoma de Nuevo León, Facultad de Enfermería; Escuela de Enfermería del, Monterrey, Nuevo León, México.

Maestria en ciencias, Instituto Mexicano del Seguro Social ; boctorado en Ciencias de Enfermería
} 


\section{INTRODUCCIÓN}

La Organización Mundial de la Salud reportó que en el mundo se han registrado más de 347 millones de personas con diabetes, además calcula que por esta causa en el 2012 fallecieron 1,5 millones de personas, otro dato importante es que más del $80 \%$ de las defunciones reportadas por esta patología se han registrado en países de ingresos bajos y medios. Esta patología puede controlarse llevando un adecuado autocuidado (1).

Las prácticas de autocuidado son las actividades que las personas realizan, para su propio beneficio y lograr el mantenimiento de la vida, un funcionamiento saludable y un continuo desarrollo personal y de bienestar. En el caso de la persona con diabetes tipo 2 (DT2) se refiere al auto-monitoreo, actividad física, alimentación, revisión de pies y adherencia al tratamiento, entre otros. Algunas de las premisas que maneja Orem sobre el autocuidado, son que cada persona debe realizar diariamente un mínimo de actividades dirigidas hacia sí mismo para continuar su existencia como organismo con una vida racional (2).

Cuando una persona con DT2 disminuye sus prácticas de autocuidado, incrementa el riesgo de presentar alteraciones en el control glucémico, dando como resultado niveles elevados de glucosa en sangre (3). La literatura reporta algunas barreras que bloquean el adecuado cuidado de la diabetes, entre ellas se encuentra la adherencia al régimen de autocuidado, actitudes y creencias, conocimiento, y escasos recursos financieros (4).

Una adecuada adherencia puede reducir la morbimortalidad, discapacidad y con ello mejorar su calidad de vida y economizar los costos destinados a la atención de la salud. Por otra parte las personas con diabetes desarrollan actitudes y creencias respecto a que es la diabetes y el tratamiento que en la mayoría de las ocasiones distorsionan la necesidad e importancia de llevar adecuadas prácticas de salud. Otro obstáculo es el recurso financiero y mayormente en población con recurso económico bajo que además no tiene un servicio de salud.

El objetivo del estudio fue determinar diferencias de autocuidado por edad y sexo en adultos mayores con diabetes tipo 2, que acuden a la consulta de medicina familiar.

\section{MATERIAL Y MÉTODOS}

Diseño y población de estudio.
Se realizó un estudio transversal en adultos de 60 o más años, con diabetes tipo 2, que asistieron a la consulta en una Unidad de Medicina Familiar (UMF) de una institución de seguridad social en Monterrey, México; y que no asistieran al programa de atención para control de diabetes. El cálculo de tamaño de muestra $(\mathrm{n}=88 ; 44$ para cada grupo mujeres/hombres) se realizó esperando una diferencia de medias de 0,6 puntos en el cuestionario de autocuidado a favor del grupo de menos edad y del género femenino. Se realizó en Epidat 3.1 (5) con un nivel de confianza del $95 \%$ y una potencia del $80 \%$, finalmente se encuestaron a 109 adultos mayores (63 y 46 para cada grupo). Estos fueron seleccionados de forma aleatoria simple, en el turno matutino de la unidad médica. Lo que se realizó con apoyo de la agenda electrónica controlada por la asistente médica del consultorio en el que aparece el nombre de los pacientes y su diagnóstico.

\section{Instrumento de medición.}

Se aplicó el Resumen de Actividades de Autocuidado en Diabetes (SDSCA, por sus siglas en inglés), instrumento que ha reportado una alfa de Cronbach de 0,68 (6). Contiene reactivos que miden las prácticas de autocuidado de la diabetes en los últimos siete días entre las que se encuentran: alimentación con cinco reactivos, ejercicio con dos, el automonitoreo con dos, cuidado de los pies con cuatro y adherencia a medicamentos con dos, todos con opción a ocho respuestas que corresponden al número de días de la semana: $0,1,2,3,4,5,6$ y 7 , y que al final promedian los reactivos con puntajes de días de prácticas de autocuidado en forma general y para cada subescala de 0 a 7; a mayor promedio de días, mayores prácticas de autocuidado realizadas por el paciente con DT2. Además cuenta con dos reactivos que evalúan el estado de fumador con opción a dos respuestas; no y sí (7). Para fines de éste estudio el autocuidado se dicotomizó 1$)<5$ días $=$ sin prácticas de autocuidado y 2 ) $\geq 5$ con prácticas de autocuidado.

\section{Procedimientos}

Al inicio del día y previa presentación con autoridades de la UMF, se asignaron en forma consecutiva los consultorios que ese día se revisarían; se contó con personal capacitado previamente (pasantes de enfermería) para la aplicación de los instrumentos los cuales individualmente identificaron al participante con su nombre en la sala de espera, antes de la consulta médica, se le explicó el propósito del estudio y en caso de aceptar participar en éste se realizaron las mediciones de lápiz y papel en un área 
específica para ello y solicitada previamente a la Dirección de la UMF; dichas actividades se llevaron a cabo sin interferir con la consulta médica. Al terminar de responder, se proporcionó información acerca del autocuidado en DT2 impresa en un tríptico.

\section{Consideraciones éticas}

El presente estudio consideró las disposiciones dictadas en el Reglamento de la Ley General de Salud en Materia de Investigación, vigentes en el país (8). Fue aprobado por los Comités de Ética e Investigación de la Facultad de Enfermería, de la Universidad Autónoma de Nuevo León.

\section{Plan de análisis}

Se utilizó estadística descriptiva (frecuencias simples y relativas, medidas de dispersión e IC95\%), U de Mann-Whitney y diferencia de proporciones.

\section{RESULTADOS}

\section{Características sociodemográficas y clínicas de la po- blación}

Los adultos mayores presentaron una edad promedio de $69,4( \pm 6,4)$ años, 63 fueron mujeres $(57,8 \%)$ que presentaron en promedio una escolaridad de 5,2 $( \pm 3,7)$ años; y respecto a la ocupación: $55(50,5 \%)$ se dedicaban al hogar, $45(41,3 \%)$ eran jubilados o pensionados y solo 9 $(8,2 \%)$ reportaron trabajar.

Por otra parte, el promedio de tiempo de diagnóstico con la DT2 fue de 12,24 ( \pm 9.9$)$ años, 50 de los adultos mayores $(55,9 \%)$ reportaron tener co-morbilidad, la más común fue la hipertensión arterial para 43 adultos $(39,4 \%)$; además, $34(31,2 \%)$ comentaron tener alguna complicación, la más común fue la retinopatía $(22,9 \%)$.

Tabla 1. Días a la semana de prácticas de Autocuidado en 109 adultos mayores en una institución de seguridad social.

\begin{tabular}{lccccc}
\hline & & & & \multicolumn{2}{c}{ IC95\% } \\
& & $D E$ & $M d$ & Inferior & Superior \\
\hline Dieta & 3,75 & 1,26 & 4,0 & 3,51 & 3,99 \\
Ejercicio & 2,90 & 2,60 & 2,5 & 2,41 & 3,40 \\
Automonitoreo & 3,83 & 0,82 & 3,5 & 3,67 & 3,99 \\
Cuidado de pies & 6,27 & 1,34 & 7,0 & 6,02 & 6,53 \\
Medicamentos & 6,68 & 1,01 & 7,0 & 6,49 & 6,88 \\
\hline
\end{tabular}

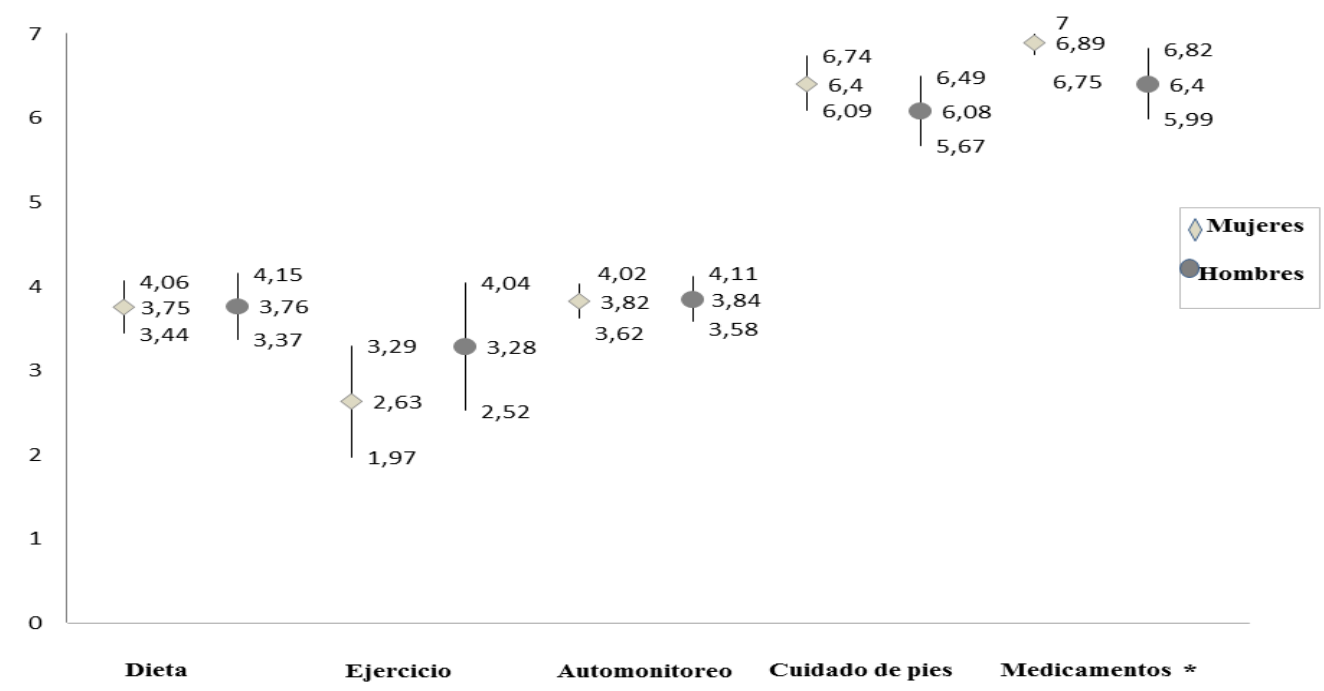

Nota: * $\mathrm{p}<0.05$

Figura 1. Días a la semana de prácticas de Autocuidado en 109 adultos mayores en una institución de seguridad social por género. 




Figura 2. Días a la semana de prácticas de Autocuidado en 109 adultos mayores en una institución de seguridad social por edad.

\section{Autocuidado del adulto mayor con DT2.}

Sólo $36(33,0 \%)$ de los adultos mayores tuvieron prácticas de autocuidado, las realizaban en promedio 4,69 $( \pm$ 0,7; IC95\% 4,55, 4,82) días. En la tabla 1, se observan las subescalas del autocuidado, las que se mencionaron efectuaban con más frecuencia a la semana fueron cuidado de pies y medicamentos. Al analizar por género se observó que el $28,6 \%$ de las mujeres presentaron prácticas de autocuidado, mientras que el $39,1 \%$ en hombres $(p>0,05)$. No se presentaron diferencias en los días que las realizaban (mujeres 4,7 $\pm 0,6$ vs hombres $4,7 \pm 0,8, p$ $>0,05)$; las mujeres presentaron mayor promedio de días de autocuidado en medicamentos (Figura 1). El 34,9\% de los adultos mayores de 60 a 69 años presentaron prácticas de autocuidado, mientras que en los $>$ de 70 años el 30,4\%, $(p>0,05)$. Ambos grupos fueron iguales en cuanto a los días de práctica de autocuidado ( 60 a 69 años $4,8 \pm 0,7$ vs 70 o más años $4,6 \pm 0,7, p>0,05)$; los adultos de menos edad presentaron mayor promedio de días de autocuidado respecto a la dieta (Figura 2).

\section{DISCUSIÓN}

Las características sociodemográficas de la población estudiada fueron similares a lo reportado en la literatura
$(3,9,10,11,12)$. En cuanto a las características clínicas el estudio coincide con el de Fabián et al.(11),quienes reportan a la retinopatía como la complicación con mayor frecuencia ya que en el presente estudio, tres de cada 10 personas reportaron padecerla. Es importante señalar que el estudio de Fabián et al. fue realizado en un hospital de tercer nivel en donde las complicaciones están diagnosticadas en los pacientes y en el actual sólo se le preguntó al participante.

Las prácticas de autocuidado se mostraron disminuidas ya que solamente una tercera parte de la población cumplía con los criterios adecuados, esto coincide con lo que se reporta en la ENSANUT (13) donde se menciona que solo la cuarta parte de las personas diagnosticadas con diabetes realizan prácticas para llevar un adecuado control. A las actividades de cuidado que le brindan mayor importancia son los medicamentos y cuidado de los pies, similar a lo mencionado en el artículo de Hernández-Cortés et al., (14) donde menciona que los adultos mayores le dan mayor peso a la medicación que otros factores de cuidado a la salud.

Respecto al cuidado de los pies los resultados coinciden a lo señalado por Hailu (15), esto puede verse influenciado por la educación que los pacientes reciben respecto a 
los cuidados de los miembros inferiores, ya que puede ser un tema nuevo e interesante para esta población. Sin embargo, se sabe que en nuestro medio la población con diabetes tipo 2 tiene muchas creencias al respecto por lo que en especial con el cuidado de los pies presentan más miedo a perder alguna parte de su cuerpo que a presentar obesidad por ejemplo; situación que deberá ser estudiada y se podrán realizar intervenciones dirigidas al cambio de éstas. Se ha reportado que actividades de autocuidado de forma independiente no contribuyen al control glucémico, más bien tienen que realizarse en conjunto como llevar una dieta adecuada y la realización de actividad física (15),estas actividades en este estudio recibieron los puntajes más bajos.

\section{CONCLUSIONES}

Aunque la diferencia no fue significativa mayor proporción de hombres realizan prácticas de autocuidado, esto es diferente a lo esperado ya que en la población mexicana son las mujeres quienes tienen mayor participación en la asistencia a programas educativos y de control a la salud; esto se ve reflejado en los días que toman su medicación. Los adultos de menor edad presentan mayores prácticas de autocuidado esto puede deberse al grado de independencia que ellos tienen comparado con las personas de mayor edad; al igual que en los días que se adhieren a la dieta. Considerando que la esperanza de vida va en incremento pero no la salud de los adultos mayores, este tipo de trabajos son importantes para conocer las prácticas de autocuidado en esta población. Además permitirá diseñar intervenciones de enfermería dirigidas a prevenir complicaciones de la diabetes tipo 2 tratando de evitar discapacidades y con esto aportar al envejecimiento exitoso a pesar de la enfermedad.

\section{REFERENCIAS BIBLIOGRAFICAS}

1. Organización Mundial de la Salud. Diabetes, Nota descriptiva $\mathrm{N}^{\circ} 312$. Ginebra: Organización Mundial de la Salud;6 de junio del 2014. Disponible en http://www.who. int/mediacentre/factsheets/fs312/es/

2. Orem DE. Modelo de Orem: Conceptos de Enfermería en la Práctica. Barcelona. España: Masson-Salvat; 1993.

3. Castro G, Tovar J, Mendoza U. Trastorno depresivo y control glucémico en el paciente con diabetes mellitus 2. Rev Med Inst Mex Seguro Soc. 2009; 47:377-382.

4. Nam S, Chesla C, Stotts N, Kroon L, Janson SL. Barriers to diabetes management: Patient and provider factors. Diabetes Res Clin Pract. 2011; 93: 1-9.

5. Dirección Xeral de Saúde Pública, Xunta de Galicia ; Organización Panamericana de la Salud;Instituto Superior de Ciencias Médicas de la Habana. EPIDAT 3.1, Programa para análisis epidemiológico de datos tabulados La Habana, Cuba: Instituto Superior de Ciencias Médicas de la Habana; 2003.

6. McEwen V, Pasvogel A. The validity and reliability of a Spanish version of the summary of diabetes self-care activities questionnaire. Nurs Res. 2008;57:101-6.

7. Toobert D, Hampson S, Glasgow R. The summary of Diabetes Self-Care Activities Measure. Diabetes Care. 2000;23: 943-950.

8. Secretaría de Salud. Reglamento de la Ley General de Salud en Materia de Investigación para la Salud. México: Secretaría de Salud; 1987. (Citado el 20 de Abril del 2014) Disponible en http://www.salud.gob.mx/unidades/cdi/nom/ compi/rlgsmis.htm

9. Colunga C, García J, Salazar J, Ángel M. Diabetes Tipo 2 y Depresión en Guadalajara, México. Revista de Salud Pública. 2008; 10: 137-149.

10. Egede L, Ellis Ch, Grubaugh A. The Effect of depression on self-care behaviors and quality of care in a national simple of adults with diabetes. General Hospital Psychiatry, 2009, 31, 422-427.

11. Fabián M, García M, Cobo A. Prevalencia de síntomas de ansiedad y depresión en pacientes con diabetes mellitus tipo 2 y su asociación con el tipo de tratamiento, complicaciones de la diabetes y comorbilidades. Medicina Interna de México. 2010; 26: 100-108.

12. González J, Safren S, Cagliero E, et al. Depression, SelfCare and Medication Adherence in Type 2 Diabetes. Diabetes Care. 2007, 30, 2222-2227.

13. Sistema Nacional de Encuestas de Salud. Encuesta Nacional de Salud y Nutrición: Resultados Nacionales. Mexico DF:Sistema Nacional de Encuestas de Salud;2012, Recuperado de http://ensanut.insp.mx/ informes/ENSANUT2012 ResultadosNacionales.pdf

14. Hernández-Cortés PL, Morales-Martínes G , LopézRamirez EO, Salazar-González B. Systematic thinking underlying people with diabetes' beliefs on health outcomes: An information integration theory approach. Advances in Aging Research. 2014; 3: 310-317

15. Hailu E, Mariam W, Belachew T, Birhanu Z. Self-care practice and glycaemic control amongst adults with diabetes at the Jimma University Specialized Hospital in south-west Ethiopia: A cross-sectional study. Afr J Prim Health Care FamMed. 2012; 4(1): 1-6.

\section{Correspondencia:}

Correspondencia:

Flores-Pérez Martha Nelly

Correo electrónico: nellydeloredo@hotmail.com

Fecha de Recepción: 03 de Agosto del 2015.

Fecha de aceptación: 09 de Noviembre del 2015. 